\title{
El concepto de desarrollo sustentable treinta años después ${ }^{1}$
}

\author{
Guillermo FOLADORI* \\ Humberto TOMMASINO**
}

\begin{abstract}
RESUMO
Neste artigo fazemos uma análise da evolução do conceito de desenvolvimento sustentável nos últimos trinta anos. Nosso principal argumento é que existem três eixos a partir dos quais podem-se agrupar os diferentes enfoques. O primeiro restringe o conceito de desenvolvimento sustentável à sustentabilidade ecológica. O segundo, soma à sustentabilidade ecológica uma social, mas somente como ponte para a análise da sustentabilidade ecológica. Um terceiro eixo considera o desenvolvimento sustentável como a coevolução da sustentabilidade ecológica e social.
\end{abstract}

Palavras-chave: Desenvolvimento Sustentável, Meio ambiente

\begin{abstract}
In this article we analyze the evolution of the concept of sustentable development during the last thirty years. Our main argument is that there exist three approaches. One limits the concept of sustainable development to the ecological sustainability. The other adds to the ecological sustainability a social sustainability, but only as a bridge to analyze the ecological sustainability. A third one considers sustainable development, as been the coevolution of an ecological and a social sustainability.
\end{abstract}

Key-words: Sustentable Development, Environment

1 Los autores agradecen los comentarios de Clovis Ultramari.

* Professor Visitante. Doutorado em Meio Ambiente e Desenvolvimento. UFPR. E-mail: fola@ cce.ufpr.br.

** Doutorando. Doutorado em Meio Ambiente e Desenvolvimento. UFPR. E-mail: toma@ barigui.ufpr.br. 


\section{Introducción}

En este artículo reseñamos la evolución del concepto de desarrollo sustentable en las tres últimas décadas del siglo XX a partir de las conferencias e informes internacionales sobre medio ambiente, así como de las principales corrientes del pensamiento ambientalista.

Coincidimos con las críticas de Lélé (1991) quien señala que la discusión sobre desarrollo sustentable debe aclarar los conceptos de sustentabilidad ecológica y sustentabilidad social, que son la causa de la mayoría de los desacuerdos.

Nuestro principal argumento consiste en mostrar que existen dos grandes concepciones sobre la sustentabilidad social. Una, que es la predominante y que llamaremos de sustentabilidad social limitada, utiliza la sustentabilidad social como puente para llegar a la sustentabilidad ecológica. Para ésta las soluciones son básicamente técnicas. La otra, que llamaremos de coevolución sociedad-naturaleza, considera que los problemas sociales son también parte del desarrollo insustentable; y las soluciones deben ser consideradas tanto desde un punto de vista técnico, como social. Para que el lector pueda visualizar las distintas posiciones, autores e instituciones sobre la sustentabilidad ecológica y la sustentabilidad social incluímos un diagrama en forma de continuo con ejemplos y referencias bibliográficas.

Las diferencias en el entendimiento de lo que es, o debe ser, la sustentabilidad son también fruto de la evolución de la discusión, por ello comenzamos con una referencia histórica - que puede ser ampliada en el anexo- de los eventos que marcaron el rumbo de la discusión.

\section{Etapas en la evolución del concepto de desarrollo sustentable}

El trabajo, como actividad que transforma la naturaleza con el propósito de crear objetos útiles y la consecuente acumulación de información a través del tiempo, es la esencia de la naturaleza humana (Foladori, 1999). Esta actividad conlleva, asimismo, la modificación de la propia sociedad humana. De allí que los grados de transformación de la naturaleza van ligados a diferentes estructuras sociales y relaciones de producción. El ser humano ha afectado la naturaleza de forma radical desde sus épocas más primitivas (Simmons, 1993; Foster, 1994). Y también reconoció y se preocupó desde tiempos antiguos por el daño que causaba a su medio ambiente.

No obstante, el siglo XX se caracterizó por transformaciones mas profundas sobre el ambiente, tanto en cantidad como en calidad. En cantidad y amplitud porque muchos de los efectos sobre el medio pasaron de ser regionales o locales a alcanzar una escala planetaria, como el calentamiento global, la disminución de la capa de ozono o la pérdida de la biodiversidad. En calidad o profundidad porque el avance de la química permitió la ruptura de lazos y combinaciones de elementos nuevos que generaron efectos de largo plazo sobre el medio ambiente. De allí que la preocupación por el medio ambiente surgida durante la década de los sesenta en los países desarrollados tenga un propósito diferente a la preocupación de épocas y periodos históricos anteriores. La diferencia consistió, básicamente, en relacionar el deterioro ambiental con el desarrollo, mostrando que el desarrollo por sí mismo - y contra la idea que se tenía hasta los cincuenta - no daba cuenta de un equilibrio con el medio ambiente, y tampoco lograba la equidad al interior de la sociedad humana.

A principios de los años setenta del siglo $X X$ ya se podían visualizar cuatro posiciones respecto a la problemática ambiental. Por un lado, estaban los catastrofistas para quienes la continuación del ritmo de crecimiento llevaría, en el correr del siglo XXI, a una catástrofe ecológica y humana, debido principalmente a la escasez de recursos naturales. Dentro de esta línea, el informe para el Club de Roma Los límites del crecimiento (1972) constituyó un mojón en la discusión. Por otro lado, estaban los tecnócratas optimistas, los cornucopianos, que defendían la inagotable fuente de la naturaleza y el eficaz mecanismo del mercado como regulador del uso de los recursos; véase, por ejemplo The Resourceful Earth (1984). Para estos no se vislumbra crisis ambiental alguna. También estaban los ecologistas a ultranza, que frente a la crisis de la sociedad industrial abogaban por una "vuelta al pasado" en una 
reivindicación romántica de la vida rural en pequeñas comunidades autosustentables. Para deslindarse de estas posiciones surgieron una serie de planteos tendientes a interpretar la crisis ambiental y ofrecer una salida "viable" al capitalismo. El término "ecodesarrollo" y luego el de "desarrollo sustentable" constituyen el centro de la discusión.

De acuerdo con Sachs (1994), la concepción del "eco-desarrollo" (término que luego cambió por desarrollo sustentable) comienza a gestarse a partir de la reunión de ONU-EPHE (1972), que fue preparatoria para la Conferencia de las Naciones Unidas sobre el Medio Ambiente Humano en Estocolmo (1972). ${ }^{2}$ Allí se rechazaron las "visiones reduccionistas de la ecología intransigente y del economismo restricto" y se preconizó una "via intermediaria" entre el "pesimismo malthusiano", preocupado por el agotamiento de los recursos y el "optimismo de los teóricos de la abundancia" que creen en las soluciones tecnológicas (Sachs, 1994, p. 47). En este encuentro se resaltó que los problemas ambientales y de desarrollo eran compatibles y debían tener una alternativa común.

Para Sachs, ecodesarrollo es un "...concepto que podemos definir como un desarrollo deseable desde el punto de vista social, viable desde el punto de vista económico y prudente desde el ecológico" (Sachs, 1980, p. 719).

Los principios básicos del concepto son:

\begin{abstract}
“i) satisfação das necessidades básicas, ii) solidariedade com as gerações futuras, iii) participação da população envolvida, iv) preservação dos recursos naturais e meio ambiente em geral, v) elaboração de um sistema social garantindo emprego, segurança social e respeito a outras culturas, vi) programs de educação, vii) defesa da separação dos paises centrais e perifericos para garantir o desenvolvimento dos últimos (SACHS, 1994:52)”.
\end{abstract}

Los aspectos que abarca el concepto son: a) viabilidad social: que tiene como centro la construcción de una civilización con mayor justicia en la repartición de riquezas y rentas, «tendo como objetivo a redução da distância no nível de vida entre providos e deserdados»; b) viabilidad económica: «tornada possivel pela repartição e pela gestão mais eficiente dos recursos, e por um fluxo regular de investimentos públicos e privados»; c) viabilidad ecológica: que sostiene puede ser alcanzada si se toman la siguientes medidas: -intensificación de la explotación potencial de los recursos de los diversos ecosistemas, causando el menor daño posible en los sistemas que mantienen la vida, -limitación del consumo de combustibles fósiles y otros recursos no renovables, reduciendo el volumen de residuos y contaminación y economizando y reciclando energía, «incitar os ricos, em escala nacional e individual, a limitar voluntariamente o consumo de bens materiais», - intensificar la investigación sobre tecnologías limpias, - definir reglas para la protección del medio ambiente (combinación de instrumentos económicos, jurídicos y administrativos); d) viabilidad espacial: con el objetivo de equilibrar las poblaciones de ciudad y campo y una mejor repartición de la actividad económica en todo el territorio; e) viabilidad cultural: que implica que el ecodesarrollo asuma una pluralidad de soluciones locales específicas para cada ecosistema, cada cultura y cada situación (Sachs, 1994, p. 52).

En 1972 se realiza en Estocolmo, Suecia, la primera Conferencia de la Organización de Naciones Unidas sobre el Medio Ambiente y el Hombre. El resultado fue una declaración donde se abordan los principales problemas relacionados con el medio ambiente: industrialización, explosión demográfica y crecimiento urbano. Se proclama "el derecho de los seres humanos a un medio ambiente sano y el deber de protegerlo y mejorarlo para las futuras generaciones". Como resultado se crea el UNEP (Programa de Naciones Unidas para el Medio Ambiente), y la WCED (Comisión Mundial para el Medio Ambiente y el Desarrollo) que emitirá su

2 Según Caldwell, et al (1984) el concepto de desarrollo ecológicamente sustentable estuvo presente implícitamente en la Conferencia Intergubernamental de Expertos en las Bases Científicas para el uso racional y la Conservación de la Biósfera ("Biosphere Conference") realizada en París en setiembre de 1968, así como en la Conferencia sobre Aspectos Ecológicos del Desarrollo Internacional realizada en diciembre de 1968 en las cercanías de Washington DC y organizada por The Conservation Foundation y el Center for the Biology of Natural Systemas de Washington University. 
informe sobre el medio ambiente y el mundo en 1987 (informe Brundtland). El espíritu general de la declaración partía de la base de que con tecnologías limpias en los países desarrollados; y transferencia de recursos financieros y técnicos para el Tercer Mundo, junto a políticas de control de la población, podían solucionarse los problemas. Se vislumbraron contradicciones entre los países ricos que pretendían controlar la producción y la explosión demográfica y los pobres que veían la necesidad del desarrollo. ${ }^{3}$

En 1974 se realiza la Conferencia de Cocoyoc, donde el Programa de Naciones Unidas para el Medio Ambiente (UNEP) y la Conferencia de Naciones Unidas sobre Comercio y Desarrollo (UNCTAD) emiten una declaración inspirada en el concepto de ecodesarrollo. A pesar de que la reunión debía encarar problemas fundamentalmente de contaminación, de hecho discutió y estableció una clara relación entre el modelo industrialista de desarrollo y sus efectos sobre el medio ambiente. La cuestión de los límites físicos al desarrollo fué colocado explícitamente.

Bajo la dirección de la Sra. Brundtland se da a conocer, en 1987, el informe de la Comisión Mundial para el Medio Ambiente y Desarrollo (WCED, 1987), un grupo independiente pero creado a solicitud de la Asamblea General de las Naciones Unidas para elaborar una "agenda global para el cambio". En dicho informe, Our Common Future, se examinan los problemas más críticos en torno al desarrollo y el medio ambiente y se indican propuestas de solución. A partir de allí se difunde el término de desarrollo sustentable, como "aquel que responde a las necesidades del presente de forma igualitaria pero sin comprometer las posibilidades de sobrevivencia y prosperidad de las generaciones futuras"; y se establece que la pobreza, la igualdad y la degradación ambiental no pueden ser analizados de manera aislada. El documento coloca a la pobreza como una de las causas (y consecuencias) de los problemas ambientales.

En 1992 tiene lugar, en Rio de Janeiro, la Conferencia de las Naciones Unidas sobre el Medio Ambiente y Desarrollo (CNUMAD), la conocida por Cumbre de la Tierra; llamada a elaborar estrategias y medidas con el fin de detener y revertir la degradación ambiental, bien como promover el desarrollo sustentable respecto del medio ambiente. Se emiten una serie de declaraciones, dentro de ellas destacan: a) la convención sobre cambio climático; una recomendación para estabilizar las emisiones de $\mathrm{CO}_{2}$ para el año 2000 a niveles de 1990. Un recomendación sin compromiso alguno, que mostró cómo una cuestión a primera vista de interés global, tiene en realidad intereses contrapuestos cuando se analiza con mayor detenimiento. Los EEUU no estuvieron dispuestos a reestructurar su industria como tampoco a disminuir su nivel de consumo energético y los países árabes petroleros tampoco se vieron dispuestos a disminuir su producción y ventas. b) la convención sobre la biodiversidad; que reconoció la soberanía de cada país respecto de su patrimonio biogenético. No fue firmada por los EEUU, que presionado por las transnacionales farmacéuticas, pretendía patentes para los laboratorios que descubrían nuevas drogas o usos de especies vivas. c) la declaración de principios sobre el manejo, la conservación y desarrollo sustentable de todos los bosques; terminó siendo una intención de frenar el desmatamiento. Y, la agenda 21 ; que pretende ser un plan de acción para el próximo siglo, donde se destacan 31 puntos esenciales y se resuelve que el Banco Mundial sea el encargado de orientar los fondos (Guimarães, 1992).

El anexo 1 ilustra las principais caracteristicas y algunos comentarios de las conferencias o informes que constituyeron mojones claves en la evolución del concepto de desarrollo sustentable.

3 En América Latina, desde la década de los setenta, la Comisión Bariloche y la CEPAL realizan importantes investigaciones y propuestas de modelos alternativos de desarrollo. Se planteó, por ejemplo, la renuncia a un crecimiento ilimitado, el freno a la exportación masiva de recursos naturales, y se señaló que el objetivo del desenvolvimiento debiera ser la calidad de vida. Se señaló que la sustentabilidad debía cumplir tres requisitos: no empobrecer a un grupo al tiempo que se enriquece otro, no degradar la diversidad y la productividad biológica ni los procesos ecológicos y sistemas vitales esenciales, y aumentar la capacidad de self-reliance (Herrera, et al., 1971; Sunkel, et al., 1986). 


\section{Problemas y contradicciones en el concepto de desarrollo sustentable}

Sustentabilidad, en términos literales, significa continuación a través del tiempo:

"Taken literally, sustainable development would simply mean `development that can be continued - either indefinitely or for the implicit tim period of concern" (Lélé, 1991, p. 608-609).

Cuando este término se agrega al de desarrollo se supone que lo que debe sustentarse es el desarrollo. Pero, como bien anotan Barbier (1987) y Lélé (1991) entre otros, el concepto de desarrollo es sumamente vago, se presta a las más variadas definiciones, y ha ido evolucionando a través del tiempo. No obstante, y como señala Harborth (1991), hay dos grandes objetivos que el concepto de desarrollo sustentable pretende alcanzar:

The concept of sustainable development as a goal presumes two things: first, it implies that there are also development goals and paths - of course, those which are pursued in practice are meant - which cannot endure in the long run, because they threaten sooner or later to destroy the earth's ecological bases. Second, 'development' is by no means abandoned as a goal, and if the concept is not to be self contradictory, this means that a different development from that pursued up to now, an ecologically and socially sustainable development, is conceivable and practical (Harbort,1991, p. 9).

Pero, hay diferentes maneras de concebir estos objetivos. Para ordenar la gran diversidad de concepciones sobre el desarrollo sustentable es necesario realizar un agrupamiento - que siempre es forzado-. Creemos que las diversas posiciones pueden ser reducidas a tres grandes ejes hacia los cuales los diferentes autores se ven más o menos atraídos: aquellos para quienes la sustentabilidad es exclusivamente ecológica; aquellos para quienes la sustentabilidad es ecológica y social pero donde la parte social es un vehículo para llegar a la sustentabilidad ecológica (sustentabilidad social limitada); y aquellos para quienes la sustentabilidad debe ser realmente social y ecológica en forma de coevolución (coevolución sociedad-naturaleza).

Como puede apreciarse, en los tres ejes o grupos hay dos elementos en juego: la sustentabilidad ecológica y la sustentabilidad social. Efectivamente, la discusión sobre el desarrollo sustentable contuvo, desde su origen, dos tipos de preocupaciones. ${ }^{4}$ Una estrictamente ecológica, ligada a la depredación de recursos, al aumento de la contaminación y a la pérdida de valores "ecológicos" como la biodiversidad, los paisajes y el medio ambiente de vida en general. Una segunda preocupación -la sustentabilidad social- estuvo ligada al tema de la pobreza. De cómo la pobreza genera -al tiempo que también es resultado de-deterioro ambiental, tanto porque los pobres no tienen recursos para una estrategia productiva de largo plazo, como porque el crecimiento poblacional implica una mayor presión sobre los ecosistemas. La pobreza como problema ambiental no se restringe a los pobres propiamente dichos, sino que sus efectos alcanzan también a los ricos, como en el caso de las altas tasas de natalidad de los pobres que incrementan la población mundial y exigen mayores recursos. Estos dos "contenidos" del concepto de desarrollo sustentable ecológico y social- dieron lugar a considerar que había dos sustentabilidades: una ecológica, la otra social. Lélé (1991) muestra esta distinción y resalta cómo el concepto de sustentabilidad social siempre fue vagamente definido por los autores; algunos hablan de valores, otros de instituciones, otros de niveles de vida, etc. Esta indefinición dió lugar a dificultades para operacionalizar el concepto de desarrollo sustentable. Por ello, para Lélé, "Differentiating between ecological and social sustainability could be a first step toward clarifying some of the discussion" (Lélé, 1991, p. 615).

Para avanzar en esta distinción consideramos necesario mostrar que por detrás del concepto de sustentabilidad social hay, de hecho, dos posturas diferentes. De allí que hemos separado el segundo y tercer grupo según cómo conciben la sustentabilidad social.

4 En realidad se podría decir que son tres -y no dos- preocupaciones. También es una preocupación la faceta "económica". No obstante, lo económico está directamente ligado al desarrollo, mientras que la "novedad" del adjetivo "sustentable" fue el enfoque ecológico y el social. 
El primer grupo lo constituyen aquellos para quienes los problemas ambientales se reducen a la depredación y contaminación del medio abiótico y del resto de los seres vivos. Su interés está en lo que se conoce como sustentabilidad ecológica. Un ejemplo puede verse en la siguiente definición de Perace (1993),

\footnotetext{
What would it mean for an economy to be managed 'sustainably'? As with the fishery and forestry example, it is both the output of the economy that need to be sustained, and the underlying resource base that gives rise to that output (Perace, 1993, p. 3).

...sustainability means making sure that substitute resources are made available as non-renewable resources become physically scarce, and it means ensuring that the environmental impacts of using those resources are kept within the Earth's carrying capacity to assimilate those impacts (Pearce, 1993, p. 4).
}

Una versión aún más restringida es la difundida en la "World Conservation Strategy", de la International Union for Nature and Natural Resources (IUCN, 1980), donde la problemática ambiental se reduce a los seres vivos exclusivamente (Khosla, apud Lélé, 1991, p. 610).

El segundo grupo lo constituyen aquellos que agregan a la sustentabilidad ecológica el tema de la pobreza (sustentabilidad social limitada), considerada ésta en forma extendida, como hambre, escasez de habitación, agua potable, sistemas de salud, etc. La pobreza que aparece en el discurso principal sobre el desarrollo sustentable (v.g. ONU, Banco Mundial) le da un sesgo social a la sustentabilidad. ${ }^{5}$ Como explica Lélé (1991) pueden haber no sólo causas técnicas de la insustentabilidad ecológica, sino también sociales:
To give a stylized example, one could say that soil erosion undermining the agricultural basis for human society is a case of ecological (un) sustainability. It could be caused by farming on marginal lands without adequate soil conservation measures - the ecological cause. But the phenomenon of marginalization of peasants may have social roots, which would then be the social causes of ecological unsustainability (Lélé, 1991, p. 610).

La pobreza es considerada en estos casos en la medida en que causa insustentabilidad ecológica. Un ejemplo aclarará esto; supongamos campesinos pobres con sistemas agrícolas de quema y roza. Aquí el problema de la insustentabilidad no es que sean pobres (aunque esto obviamente no se dice), sino que su actividad, a través de la quema, aumenta el calentamiento global y conduce a la degradación de los suelos cuando el descanso es menor al exigido por el ecosistema. Otro ejemplo: siguiendo a Malthus, los pobres se reproducen más. En este caso el problema de la insustentabilidad no es que haya más pobres (lo que tampoco se dice), sino que el aumento de población presiona sobre los recursos y aumenta los desperdicios. O sea, los pobres esto es, la sustentabilidad social- es de interés en la medida en que se constituye en un elemento que afecta la sustentabilidad ecológica. ${ }^{6}$ Por sí misma la pobreza no es un problema ambiental, lo son las consecuencias que ésta genera en el medio ambiente. Se trata de una sustentabilidad social limitada, restringida a su papel de puente para el análisis de la sustentabilidad ecológica. Es necesario, entonces, no confundir palabras como pobreza, migraciones, hambre, hacinamiento, etc. con relaciones sociales, porque lo que interesa son las relaciones técnicas entre los pobres y el uso de recursos naturales. Las relaciones sociales, que se refieren a cómo determinadas relaciones entre los seres humanos generan

5 Véase, por ejemplo, las definiciones del informe Brundtland (1987) o del Banco Mundial (1987) respectivamente:

"sustainable development is development that meets the needs of the present without compromising the ability of future generations to meet their own needs" (WCED, 1987, p. 43).

a pattern of social and structural economic transformations (i.e. 'development') which optimizes the economic and other societal benefits available in the present without jeopardizing the likely potential for similar benefits in the future (Goodland/Ledec, 1986, apud Barbier, 1987, p.105).

6 El momento histórico de reconocimiento de la in-sustentabilidad social delata su entendimiento como puente para la insustentabilidad ecológica. La sustentabilidad social limitada es una consecuencia de la preocupación por la sustentabilidad ecológica de las décadas del setenta y ochenta. Pero, ;la crisis social (pobreza, hambre, desempleo, etc.) es muy anterior! 
pobreza, desempleo, hambre, etc. no están en discusión, sino sólo sus consecuencias técnicas en la contaminación y depredación del medio.

Tanto en el primer grupo, de la sustentabilidad ecológica exclusiva, como en este segundo, de la sustentabilidad ecológica y social limitada, la concepción de la problemática ambiental es técnica. Es de relación entre el ser humano y las cosas; sean estas cosas otros seres vivos, o materia abiótica. Cuando, en este segundo grupo, se habla de sustentabilidad social el problema es también de cosas, escasez de agua potable, técnicas productivas depredadoras, falta de alimento, etc. En los dos primeros grupos los problemas ambientales pueden y deben ser resueltos mediante técnicas "sustentables": tecnologías limpias o verdes, mejor aprovechamiento de los resíduos, aumento de la productividad en el uso de los recursos naturales, cambio en el uso de recursos no renovables hacia renovables, etc. Como puede verse, se trata en todos los casos de soluciones técnicas.

El segundo grupo representa la posición "oficial" sobre el desarrollo sustentable. La que difunden los organismos internacionales como la ONU, y el Banco Mundial, o las instituciones como el World Watch Institute. Claro está que existen algunas diferencias entre ellos, no obstante, el concepto de desarrollo sustentable abarca, resumidamente, tres aspectos: a) la degradación ambiental (en gran parte causada por la pobreza pero cuyos resultados afectan a todos), b) los objetivos tradicionales del desarrollo como el aumento de la productividad para satisfacción de las necesidades básicas, ligado a métodos ambientalmente "amigables" $\mathrm{y}$, en algunos casos, c) procesos de desarrollo participatorios (Lélé, 1991).

Para el tercer grupo (coevolución sociedadnaturaleza), el medio ambiente no es sólo el entorno abiótico y de otras especies vivas, también los congéneres son parte del medio ambiente. Por lo tanto, los problemas sociales pueden generar insustentabilidad por sí mismos, más allá de que también afecten la sustentabilidad ecológica. Esto significa que la problemática ambiental debe ser analizada tanto desde la perspectiva técnica-y en esto coincide este grupo con los dos primeros-, como desde la perspectiva de las relaciones sociales. La sustentabilidad social es considerada como cuestión central, y entendida no sólo en cuanto a sus resultados técnicos como en el segundo grupo, sino en cuanto a las causas que generan la pobreza, el desempleo, el hambre, la explotación, etc.

Veamos el ejemplo del desperdicio permanente de mercancías debido a la sobreproducción, un tema nunca considerado por la sustentabilidad social limitada. La búsqueda de una mayor ganancia es una ley intrínseca de la producción capitalista. Como unas ramas generan temporalmente mayor ganancia que otras, los capitales migran hacia ellas. El resultado es la sobreproducción en unas ramas y la escasez en otras. En los siguientes ciclos los precios de las mercancías de las ramas donde hubo sobreproducción bajan y aumentan los de las ramas con subproducción, nuevamente los capitales migran hacia estas últimas. Es ampliamente conocido que el sistema capitalista se regula por la oferta y la demanda, o sea, se regula ex post. La producción de mercancías está plagada de ejemplos de quema, entierro, tirada al mar, alimentación de ganado con comida humana, o utilización como combustible de alimentos y productos que por su bajo precio no consiguen comercializarse. Hobsbawm en su Age of Extremes escribe:

“...el problema del mundo desarrollado era que producía tanto alimento que no sabía qué hacer con el excedente, y en la década de 1980 decidió plantar substancialmente menos, o entonces (como en la Comunidad Europea) vender sus 'montañas de manteca' y 'lagos de leche' abajo del costo, arruinando con eso a los productores de los países pobres. Resultó más barato comprar queso holandés en las islas del Caribe que en Holanda. Curiosamente el contraste entre excedentes de alimentos de un lado y gente hambrienta de otro, que tanto alteró al mundo durante la Gran Depresión de la década de 1930, causó menos comentarios a fines del siglo XX. Fue un aspecto de la creciente divergencia entre el mundo rico y el mundo pobre que se tornó cada vez más evidente a partir de la década de 1960" (Hobsbaw, 1995, p. 256).

La sobreproducción, que siempre existe en algunas ramas, conduce a la destrucción de mercancías, el abandono de capital fijo o de espacios naturales antes utilizados, con consecuencias deplorables para el mantenimiento de un mínimo equilibrio ecológico. Fá- 
bricas abandonadas, montañas de mercancías obsoletas en términos tal vez no de su utilidad sino de su competividad, y hasta tierras agrícolas subutilizadas una vez que los precios de mercado no permiten recuperar los costos de producción y su ganancia, son ejemplos comunes en la sociedad capitalista. Este es otro ejemplo de la insustentabilidad social cuyas causas no están en una técnica equivocada sino en el funcionamiento del mejor - mercado.

Sin ser explícito en su crítica al sistema capitalista, una idea similar, con ejemplos relacionados a la producción de mercancías supérfluas, crecimiento urbano etc., aparece en Sachs,

In the final product it may be useful to differentiate between goods and services corresponding to socially legitimized needs (that is authentic use-values), pseudouse values that are at best 'positional goods'(see Hirsh 1976) in inequality-ridden societies and non-values. The late consist of the parcel of GNP that does not serve any constructive purpose and corresponds to the rapid increase of managing and transactional costs of our societies brought about by the diseases of affluence, the accidents inherent in contemporary urban life styles and transportation modes, the environmental disruption, the diseconomies of scale of the megamachine and its twin, the megabureaucracy (Sachs, 1984, p. 211, apud Harborth, p. 24).

En estos ejemplos, que podrían extenderse significativamente, no son técnicas equivocadas que provocan insustentabilidad, sino las propias relaciones sociales capitalistas. Estas manifestaciones de insustentabilidad nunca son analizadas por los autores o instituciones que se orientan hacia los ejes que hemos llamado de sustentabilidad ecológica restringida, y de sustentabilidad social limitada. Más aún, en las diferentes mediciones de la sustentabilidad, tanto las físico-naturales, como las socio-políticas, o las económicas, no hay indicadores para medir este tipo de insustentabilidad derivado de las relaciones sociales capitalistas (Foladori, 2000).

Como señalamos en un inicio, esta caracterización en tres ejes o atractores no significa que las instituciones o autores puedan ser fácilmente encuadrados en una de las tres alternativas. La mayoría de ellos, por el contrario, tienen posiciones que se acercan a dichos ejes. Un ejemplo de la dificultad de "encasillar" autores es la siguiente cita de Barbier sobre lo que la sustentabilidad debe implicar:

Sustainable economic development is therefore directly concerned with increasing the material standard of living of the poor at the 'grassroots' level, which can be quantitatively measured in terms of increased food, real income, educational services, health-care, sanitation and water supply, emergency stocks of food and cash, etc., and only indirectly concerned with economic growth at the aggregate, commonly national, level. In general terms, the primary objective is reducing the absolute poverty of the world's poor through providing lasting and secure livelihoods that minimize resource depletion, environmental degradation, cultural disruption, and social instability (Barbier, 1987, p. 103).

Aquí pareciera que el principal problema de la sustentabilidad es la pobreza. Pero, ¿lo es por sí misma, o en función de lo que ella implica para el deterioro ecológico? Otro ejemplo, ahora tomado de una cita de Sachs,

Qué ofrece, entonces, el concepto de ecodesarrollo al planificador? En primer lugar, un criterio de racionalidad social diferente de la lógica del mercado, que se basa en los postulados éticos complementarios de la solidaridad sincrónica con la genración actual y de la solidaridad diacrónica con las generaciones futuras. El primer postulado remite a la problemática del acceso equitativo a los recursos y a la de su redistribución; el segundo obliga a extender el horizonte temporal más allá de los tiempos del economista y provoca, por tanto, una transformación de los intrumentos habitualmente utilizados para arbitrar entre el presente y el futuro (Sachs, 1980, p. 720).

¿Significa esto que las propias relaciones capitalistas son las causantes de la insustentabilidad, como diría Marx? Es claro que tanto los autores como las instituciones han modificado sus posiciones en el transcurso de los años y no es posible ni justo encasillarlos en una tipología. Debido a estas dificultades, la forma más idónea de relacionar estos tres ejes sobre la sustentabilidad con autores y declaraciones es a través de un continuo. La ubicación de diferentes grupos en el continuo es sólo tentativa e indicativa, y las referencias 
bibliográficas son ejemplificativas, pero no es posible ajustar un autor a un lugar fijo del continuo. Esto lo hacemos en el diagrama. En un extremo colocamos el concepto de sustentabilidad restringido a su consideración ecológica, en el otro extremo la sustentabilidad entendida tanto desde una perspectiva ecológica como social. Cuanto más peso se le dé a la sustentabilidad social más sobre el extremo derecho (coevolución sociedad-naturaleza) se colocan las interpretaciones. El diagrama ilustra dos ideas. La primera, que el término desarrollo sustentable es vago y se presta a variadas interpretaciones. La segunda, que las principales contradicciones y desacuerdos en torno a dicho concepto está en las relaciones interespecíficas del género humano (sustentabilidad social), antes que en las relaciones entre el ser humano y su entorno (sustentabilidad ecológica) sobre las cuales hay menos discrepancias. Para aquellos que se ubican hacia el extremo izquierdo del continuo, y dado que la sustentabilidad es exclusiva o principalmente ecológica, las soluciones son esencialmente técnicas. De hecho, todos los problemas del ser humano en relación con su entorno material son cuestiones técnicas. Para quienes se ubican hacia el extremo derecho del continuo las soluciones son tanto técnicas como sociales. Los más radicales en este sentido sostienen que las propias relaciones capitalistas conducen a un desarrollo forzosamente insustentable (Foster, 1994; Burkett, 1999). Pero estas posiciones mas volcadas sobre el extremo derecho del diagrama no son dominantes dentro de aquellos que reivindican una sustentabilidad social además de la ecológica. Para las posiciones dominantes los cambios no deben afectar las relaciones capalistas, sólo mejorarlas. El aspecto central de esta mejora está dado por la participación. Lélé (1991) explica que los postulados de justicia social y equidad que fueron característicos del concepto de "ecodesarrollo" y aún se mantuvieron en la Conferencia de Otawa (IUCN) de 1986, fueron abandonados posteriormente por las corrientes principales del desarrollo sustentable en fa- vor del término poco preciso de participación local.

En el extremo izquierdo ubicamos a los cornucopianos, tecnocentristas de mercado que consideran que no existe problema ambiental alguno y que, cuando ocurre, éste es resuelto automáticamente por el mercado. Esta posición está fuera de la discusión sobre sustentabilidad.

A continuación, hacia la derecha, y como primer ejemplo dentro del continuo del desarrollo sustentable, le sigue el grupo que denominamos de ambientalismo moderado. Esta es la versión mayoritaria y oficial sobre el desarrollo sustentable. Lo común a los dos subgrupos (a su interior) es la preocupación por corregir el capitalismo adaptándolo a los problemas que el medio ambiente causa. Para el subgrupo de más a la izquierda los problemas de sustentabilidad son sólo ecológicos, para el segundo subgrupo existe una sustentabilidad social por lograr, en la medida en que esta es puente para la ecológica - aunque esto no se diga explícitamente-.

Le sigue el grupo que llamamos de ecocentrismo. La mayoría de las posiciones de los partidos verdes y grupos ecologistas están en este grupo. Para ellos es necesario un cambio radical en el «estilo de vida», pero dicho cambio debe realizarse por la modificación de las pautas de consumo, lo cual significa -aunque no se diga- moverse también dentro de las propias relaciones capitalistas.

Por último, colocamos al marxismo. Esto requiere una aclaración, ya que debe sorprender a la mayoría de los lectores. Es comun leer que Marx tenía una concepción productivista de la naturaleza, y que sus preocupaciones nunca tuvieron un sello ecológico. De acuerdo con esta versión, no tendría sentido incluir al marxismo en este continuo de concepciones sobre la sustentabilidad. No obstante, dos recientes trabajos sobre el pensamiento de Marx en relación al medio ambiente afirman lo contrario (Burkett, 1999; Foster, 1999), y muestran no sólo la incorporación teórica de la cuestión natural en su teoría del valor y la acumulación de capital, sino también la importancia del método del materialismo histórico para pensar la actual crisis ambiental. ${ }^{7}$

7 "Anticipation of the future - real anticipation- occurs in the production of wealth in relation to the worker and to the land. The future can indeed be anticipated and ruined in both cases by premature overexertion and exhaustion, and by the disturbance of the balance between expenditure and income. In capitalist production this happens to both the worker and the land... What is shortened here exists as power and the life span of this power is shortened as a result of accelerated expenditure" (Marx, Theories of Surplus Value, T. III., 309-310, apud Burkett, 1999, p. 138). 


\section{Diagrama}

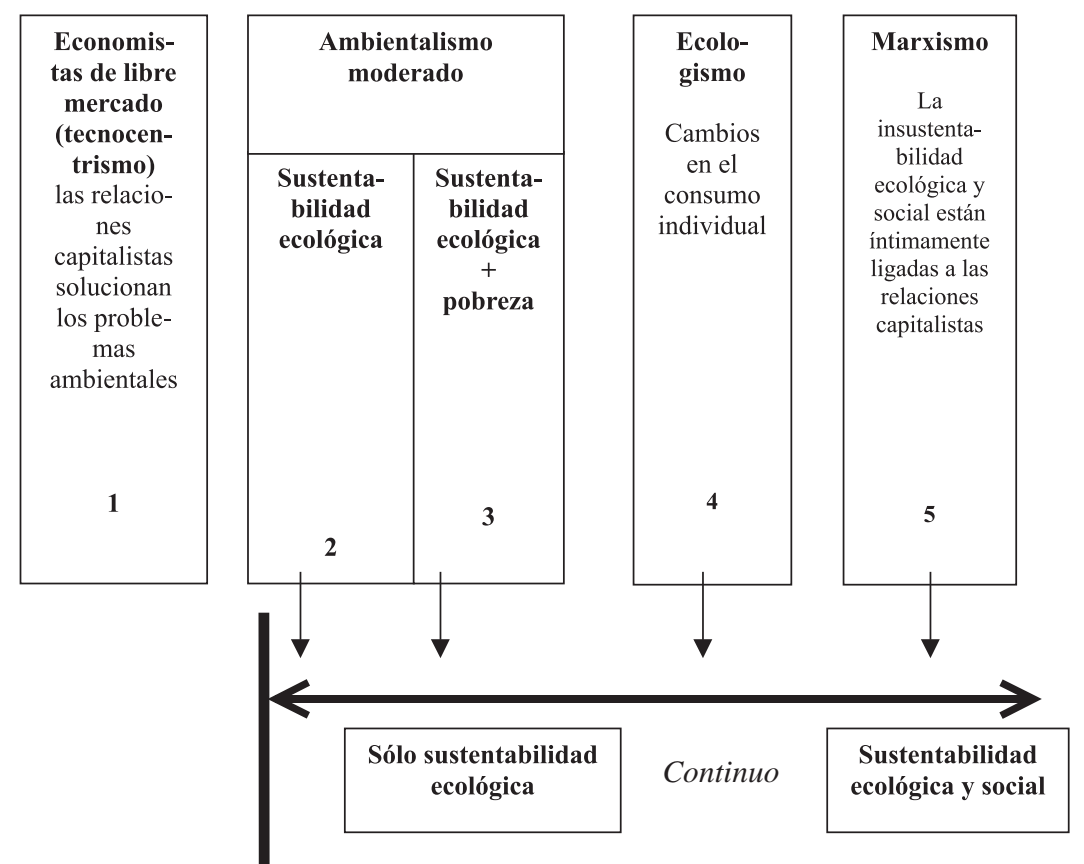

1 "We are confident that the nature of the physical world permits continued improvement in humankind's economic lot in the long run, indefinitely. ...the nature of the world's physical conditions and the resilience in a wellfunctioning economic and social system enable us to overcome such problems, and the solutions usually leave us better off than if the problem had never arisen; that is the great lesson to be learned from human history. We are less optimistic, however, about the constraints currently imposed upon material progress by political and institutional forces, in conjuction with popularly-held beliefs and attitudes about natural resources and the environment..." (Simon; Kahn, 1984, p. 3).

2 “...sustainability means making sure that substitute resources are made available as non-renewable resources become physically scarce, and it means ensuring that the environmental impacts of using those resources are kept within the Earth' $\mathrm{s}$ carrying capacity to assimilate those impacts" (Pearce \& Turner. In: Pearce, 1993, p. 4).

3 v.g. Relatorio Brundtland, Banco Mundial. "[SD has] three bases...scientific realities, consensus on ethical principles, and considerations of long-term self interest. There is a broad consensus that pursuing policies that imperil the welfare of future generations...is unfair. Most would agree that...consigning a large share of the world's population to deprivation and poverty is also unfair. Pragmatic self-interest reinforces that belief. Poverty...underlies the deterioration of resources and the population growth in much of the world and affects everyone (Repetto, 1986, p. 17, apud Lélé, 1991, p. 612).

4 "The only possible building blocks of a Greener future are individuals moving towards a Greeener way of life themselves and joining together with others who are doing the same" (Bunyard and Morgan-Greenvile, 1987, p. 336, apud Dobson, 1990, p. 141).

5 "In sum, Marx emphasizes that the same in-built tendency to endanger its own material bases is exhibited in capital's exploitation of labor power and its despoliation of natural conditions. In both cases, the overstretching of natural limits and the movement toward an eventual day of reckoning for society results from capital's ability to gain access to its immediate material requirements (exploitable labor power and conditions allowing for its exploitation), despite the ongoing vitiation of these natural conditions by capitalist exploitation intself." (Burkett, 1999, p. 139)Siguiendo estas recientes y de alguna manera novedosas interpretaciones es que lo incluímos en el diagrama. 


\section{Conclusión}

En este artículo hemos hecho una revisión del concepto de desarrollo sustentable. Mostrando que su origen se ubica en la década de los setenta y corresponde con la discusión sobre los efectos del deterioro ambiental en el desarrollo humano. El término tuvo su antecedente en el concepto de ecodesarrollo, pero fue perdiendo, frente a este último, el carácter crítico sobre las causas sociales de la insustentabilidad ecológica.

Actualmente el concepto de desarrollo sustentable tiene diferentes connotaciones, según los autores que lo utilizan. Para unos, hablar de desarrollo sustentable es referirse, exclusivamente a la sustentabilidad ecológi$c a$. Para otros, y aquí podemos ubicar las posiciones oficiales de los organismos internacionales, el desarrollo sustentable implica una sustentabilidad ecológica y una social. Sin embargo, a nuestro entender, la sustentabilidad social interesa sólo en cuanto genera sustentabilidad ecológica, y no por sí misma. En este sentido su concepto de sustentabilidad social es limitado. Por último, están quienes consideran que la sustentabilidad social es de interés por sí misma, en cuanto el desarrollo humano es una coevolución entre la sociedad y la naturaleza y no puede ser separado un aspecto de otro.

Para ejemplificar las diferentes posiciones diseñamos un diagrama que muestra un continuo, desde las posiciones más técnicas, que se interesan por la sustentabilidad sólo desde una perspectiva ecológica, hasta aquellas que relacionan los problemas de sustentabilidad ecológica y social con las propias relaciones sociales capitalistas. Como resalta Lélé (1991) una discusión y conceptualización más profunda del concepto de desarrollo sustentable es imprescindible para la aplicación de políticas apropiadas para su implementación.

A continuación de Rio se organizaron una serie de Conferencias todas ellas bajo el marco del Desarrollo Sustentable. Según Pezzoli, al conjunto se le llama Rio Cluster, y éstas fueron:

\section{Habitat II. City Summit}

1996. Midrand. UN Conference on Trade and Development

1995. Beijing. Fouth world conference on Women 1995. Copenhagen. World summit for social development

1995. New York. Migratory and Straddling Fish Stocks

1994. Cairo. Conference on Population and Development

1994. Barbados. Sustainable Development of Small Island Developing States

1994. Yokohama. World conference on Natural Disaster Reduction

1993. Vienna. World Conference on Human Rights (Pezzoli, 1997, p. 553). 


\section{Declaraciones internacionales y documentos claves para entender la evolución del concepto de Desarrollo Sustentable9}

\begin{tabular}{|c|c|}
\hline Definiciones y explicaciones & Críticas y comentarios \\
\hline $\begin{array}{l}\text { 1971 Founex (Suiza). Reunión pre parativa para la } \\
\text { Conferencia de Estocolomo (ONU, 1972). } \\
\text { Concepto de Ecodesarrollo } \\
\text { 1. Desarrollo endógeno; 2. Basado en sus propias fuerzas; } 3 . \\
\text { Tomando como punto de partida las necesidades ; } 4 . \\
\text { Promoviendo la simbiosis entre sociedad humana y naturaleza; } \\
\text { 5. Abierto al cambio institucional. (Sachs, Ignacy, 1974). }\end{array}$ & $\begin{array}{l}\text { El concepto de ecodesarrollo continuará siendo elaborado y } \\
\text { profundizado posteriormente. }\end{array}$ \\
\hline $\begin{array}{l}1972 \text { Informe del Club de Roma "Los límites del } \\
\text { crecimiento" } \\
\text { 1) Si se mantienen las tendencias actuales de crecimiento de la } \\
\text { población mundial, industrialización, contaminación ambiental, } \\
\text { producción de alimentos y agotamiento de los recursos, este } \\
\text { planeta alcanzará los límites de su crecimiento en el curso de los } \\
\text { próximos cien años. El resultado más probable sería un súbito e } \\
\text { incontrolable descenso tanto de la población como de la } \\
\text { capacidad industrial." (Meadows; Meadows, 1972, p. 40). } \\
\text { Plantea cero crecimiento tanto económico como de población } \\
\text { para una estabilidad económica y ecológica durable. }\end{array}$ & $\begin{array}{l}\text { Críticas: Universidad de Sussex. Se parte de la base de que los } \\
\text { rendimientos en la agricultura y recursos naturales son } \\
\text { decrecientes, que no habría mejora tecnológica. Planteamientos } \\
\text { neomalthusianos y neoricardianos. (Tamames, R. 1974). } \\
\text { El problema central del crecimiento son los límites físicos } \\
\text { externos. }\end{array}$ \\
\hline 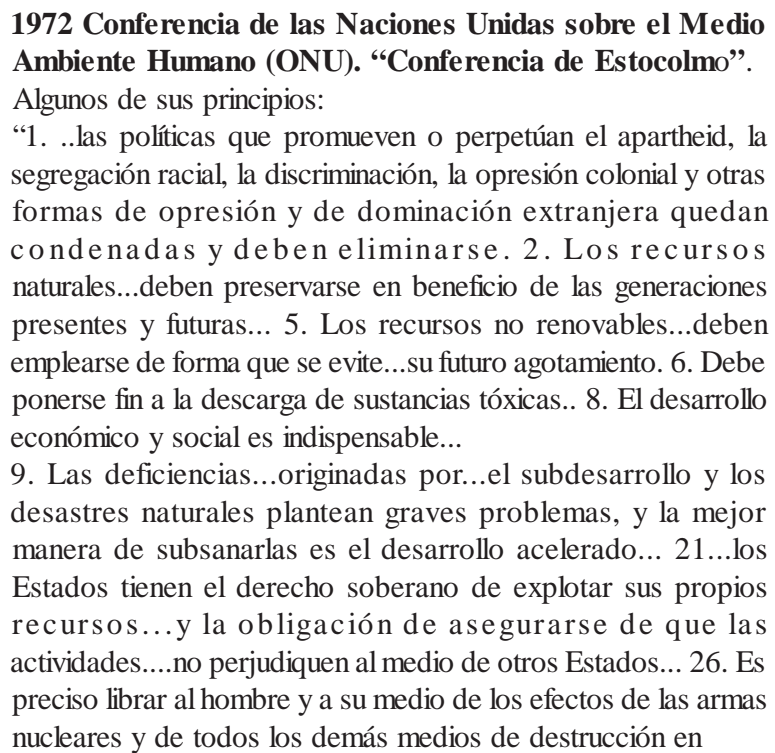 & $\begin{array}{l}\text { Strong, Maurice: "La cuestión del medio ambiente fue vista } \\
\text { inicialmente, en forma un tanto estrecha, como una enfermedad } \\
\text { de los ricos, una secuela no intencional de los procesos de } \\
\text { crecimiento económico que habían producido para ellos tales } \\
\text { niveles sin paralelo de riqueza y prosperidad. Los países en } \\
\text { desarrollo vieron esta preocupación de los ricos respecto del } \\
\text { medio ambiente como una nueva limitante potencial para su } \\
\text { propio desarrollo" (Strong, 1994:29) } \\
\text { Szekely, A; Ponce-Nava, D. : “...el problema de los patrones } \\
\text { irracionales de consumo no fue tratado en ningún principio" } \\
\text { (Székely, A; Ponce-Nava, D, 1994, p. } 315 \text { ) }\end{array}$ \\
\hline
\end{tabular}

9 La lista es parcial, y el contenido de las conferencias os informes también lo es, procurando resaltar algunos de los principales cambios en el concepto de desarrollo sustentable. 
masa..." (Declaración "Conferencia de Estocolmo". Principios. Apud Tamames, p. 197-203). Condujo a la creación del UNEP (Programa de Naciones Unidas para el Medio Ambiente) y el CMMAD (Comisión Mundial para el Medio Ambiente y el Desarrollo).

1974 UNEP (PNUMA, Programa de Naciones Unidas para el Medio Ambiente), UNCTAD (Conferencia de Naciones Unidas sobre Comercio y Desarrollo). "Conferência de Cocoyoc".

Thirty years have passed since the signing of the United Nations Charter launched the effort to establish a new international order. Today that order has reached a critical turning point. Its hopes of creating a better life for the whole human family have been largely frustrated. It has proved impossible to meet the "inner limits" of satisfying fundamental human needs. On the contrary, more people are hungry, sick, shelterless and illiterate today than when the United Nations was first se up. At the same time, new and unforeseen concerns have began to darken the international prospects. Environmental degradation and the rising pressure on resources raise the question whether the "outer limits" of the planet's physical integrity may not be at ri" (Friedmann, 1992, p. 2, apud Pezzolli, 1997, p. 551).

Plantea las siguientes hipótesis:

i) pobreza genera desequilibrio demográfico (crecimento poblacional); ii) pobreza genera degradación y contaminación de recursos; iii) los países desarrollados con alto consumo generan subdesarrollo en los países periféricos. Los países desarrollados deben disminuir su participación desproporcional en la contaminación de la atmósfera.

1975 Relatorio ¿Que Hacer? Fundación DagHammarskjold. Investigadores y políticos de 48 países, y contribuciones de la UNEP y organizaciones de la ONU

- Critica al sistema colonial y de concentración de la tierra. Exigen cambios en la estructura de propiedad de la tierra. Critican la desvatación de los paisajes

- Reivindican el "self reliance" (capacidad de autodefensa frente a cambios externos-autoconfianza).“..a harmonic relationship between society and its natural environment is full of unexplored possibilities. Linking the concept of ecological development with that of local self-reliance -each individual self-governing unit striving to solve as many of its problems as possible using the resources of its ecological system- opens up a range of perspectives..." (DHR 1975/1976, p. 34. apud, Harborth,1991, p. 23).
"The Cocoyoc meeting brought together two major strands of that period's alternative development movement: "those who had argued that priority should be given to satisfying the "basic needs" of people for food, water, and shelter rather than to simple growth-maximization, and those who were concerned with the "outer limits" of the planet's resources and its environment to sustain such growth" (Friedmann s/p, apud Pezzoli, 1997, p. $551)$.

Se utiliza el concepto de eco-desarrollo como alternativa de desarrollo (Strong, 1973).

Los escritos de mediados de los setenta, especialmente los de ecodesarrollo así como esta fundación son los que afirman que la relación de explotación de la naturaleza es un derivado de la explotación entre los hombres, y más se acercan a una crítica de las relaciones capitalistas de producción. La siguiente cita de Bennett de esta época es elocuente en ese sentido: "man's use of Nature is inextricably intertwined with man's use of Man,... remedies of destructive use of environment must be found within the social system itself" (Bennet, 1976, p. 311, apud Harborth, 1991, p. 22). 
1980 International Union for the Conservation of Nature and Natural Resources (IUCN).

"The maintenance of essential ecological processes and lifesupport systems, the preservation of genetic diversity, and the sustainable utilization of species and ecosystems' ${ }^{\prime}$ with the overall aim of achieving 'sustainable development through the conservation of living resources"" (World Conservation Startegy. IUCN, 1980, apud Barbier, 1987, p. 101). "restricted itself to living resources, focussed primarily on the necessity of maintaining genetic diversity, habits and ecological processes...It was also unable to deal adequately with sensitive or controversioal issues-those relating to the international economic and political order, war and armament, population and urbanization" (Khosla, 1987, p. 191-208. "Alternatives Strategies in Acheving Sustainable Development". Apud Lélé, 1991, p. 610). Restricta a la oferta. Considera la demanda independente y autónoma. No considera cambios en la estructura de la demanda. Sunkel, O. (1987) "Beyond the World Conservation Strategy: integrating development and the Environment in Latin-America and the Caribbean”: In: Jacobs et al., apud Lélé, 1991, p. 610)

1986 IUCN-UNEP-World Wildlife Fund. "Conferencia de Otawa".

"Sustainable Development seeks...to respond to five broad requierements: (1) integration of conservation and development, (2) satisfaction of basic human needs, (3) achievement of equity and social justice, (4) provision of social self-determination and cultural diversity, and (5) maintenance of ecological integrity".... "advancing equity and social justice...sustainable and equitable development” (Jacobs, Fardner, Munro, 1987, apud Lélé, 1991, p. 611)

1987 WCED World Commission on Environment and Development. "Informe Brundtland"

"sustainable development is development that meets the needs of the present without compromising the ability of future generations to meet their own needs" (WCED, 1987, p. 43). "We have in the past been concerned about the impacts of economic growth upon the environment. We are now forced to concern ourselves with the impacts of ecological stress -degradation of soils, water regimes, atmosphere, and forests- upon our economic prospects. We have in the more recent past been forced to face up to a sharp increase in economic interdependence among nations. We are now forced to accustom ourselves to an accelerationg ecological interdependence among nations. Ecology and economy are becoming ever more interwoven -locally, regionally, nationally, and globally- into a seamless net of causes and effects. (WCED, 1987, p. 5). 'The critical objectives which follows from the concept of SD are: (1) reviving growth; (2) changing the quality of growth; (3) meeting essential needs for jobs, food, energy, water, and sanitation; (4) ensuring a sustainable level of population; (5) conserving and enhancing the resource base; (6) reorienting technology and managing risk; (7) merging environment and economics in decision making; and; (8) reorienting international economic relations " (WCED, 1987, p. 49).
Generalidad del primer enunciado y repetición y redundancia en los siguientes fueron señalados por los reporteros Jacobs, Fardner y Munro.

Se reivindica la justicia social y la equidad -cuestiones éstas que desaparecen mas tarde v.g. Brundtland- (Lélé, 1991, p. 611).

Fue retirado el requisito de la Conferencia de Otawa de la necesidad de equidad y justicia social para el desarrollo sustentable.

Muchas organizaciones y agencias agregan a esto un noveno: "making development more participatory" (Lélé 1991, p. 611). 
1992 UNCED United Nations Conference on Environment and Development. Earth Summit. "Conferencia de Río de Janeiro".

Algunos principios:

"3. El derecho al desarrollo ...cubrir equitativamente las necesidades de desarrollo y medio ambiente de las generaciones presentes y futuras. 5. Todos los Estados...cooperarán en la tarea esencial de erradicar la pobreza. 7. Los países desarrollados reconocen la responsabilidad que tienen en la búsqueda internacional del desarrollo sustentable, en virtud de las presiones que sus sociedades imponen al mediio ambiente global...8.Los Estados deberían reducir y eliminar los patrones insostenibles de consumo y producción y promover políticas demográficas apropiadas. 10. Los temas ambientales son manejados de una mejor nanera con la participación de todos los ciudadanos... 15 . ...el enfoque precautorio se aplicará...16...promover la internalización de costos. 17 La evaluación de impacto ambiental...será llevado a cabo... (Declaración de Rio. apud Székely, Ponce-Nava, 1994, p. 306-333). "Agenda 21. Se plantea una jerarquía de las acciones que deben ir desde las directrices globales a la participación local. Se recogen una serie de iniciativas " to be put into action between now and the year 2000 It includes measures to cut energy use, protect ocean resources, promote sustainable agriculture and control toxic wastes. The Preamble of Agenda 21 begins on a foreboding but

optimistic note: "Humanity stands at a defining moment in its history. We are confronted with a perpetuation of disparities between and within nations, a worsening of poverty, hunger, ill health and illiteracy, and the continuing deterioration of the ecosystems on which we depend for our well being. However, integration of environment and devleopment concerns, and greater attention to them will lead to the fulfillment of basic needs, improved living standards for all, better protected and managed ecosystems and a safer, more prosperous future. No nation can achieve this on its own; but together we can -in a global partnership for sustainable development" (Agenda 21, apud Pezzoli, 1997, p. 552-553).
Convención sobre cambio climático: no se toman medidas urgentes, se "recomienda la estabilización de las emisiones de dióxido de carbono (CO2)a los niveles existentes en 1990. Aún asi, no fija plazos para que tal estabilización se haga efectiva" Convención sobre Biodiversidad: "El principal avance de sus 42 articulos se refiere al reconocimiento explícito de la soberanía de cada país respecto del patrimonio biogenético existente dentro de sus fronteras"

Convención sobre Bosques: “....acabó transformándose en una simple declaración de principios."

"El principal logro de la Conferencia de Rio en esa materia (nuevo orden internacional) se refiere al fortalecimiento del multilateralismo para la solución de los grandes desafíos ambientales del planeta". (Guimarães, 1992, p. 91-98).

Székely, Ponce-Nava. Uno de los aspectos principales es el reconocimiento de la responsabilidad de los países desarrollados por sus patrones de consumo (principios 7 y 8). También es un avance el reconocer la necesidad de la participación ciudadana (principio 10). (Székely, Ponce-Nava, 1994, p. 306-333)

\section{BIBLIOGRAFÍA}

BARBIER, Edward. 1987. The concept of sustainable economic development. Environmental Conservation, Switzerland. v. 14, n. 2.

BURKETT, Paul. 1999. Marx and Nature. A red and green perspective. New York: St. Martin’s Press.
CALDWELL, L; HON, L. 1984. Political aspects of ecologically sustainable development. Environmental Conservation. v. 11, n. 4, p. 299-308.

DOBSON, Andrew. 1990. Green Political Thought. London/New York: Routledge. 
FOLADORI, Guillermo. Los límites del desarrollo sustentable. Montevideo: Ediciones de la Banda Oriental/Trabajo y Capital. 1999.

2000. Sustentabilidad ambiental y contradicciones sociales". Ambiente \& Sociedade. Campinas: Nepam/Unicamp. no prelo.

FOSTER, John Bellamy. The Vulnerable Planet. New York: Monthly Review Press. 1994.

. 1999 Marx's Ecology: Materialism and Nature. New York: Monthly Review Press.

GUIMARÃES, Roberto. El discreto encanto de la Cumbre de la Tierra. Evaluación impresionista de Río 92. Nueva Sociedad. Caracas, n. 122, 1992.

HARBORTH, Hans-Jurgen. 1991. The debate about sustainable development: starting point for an environment-oriented international development policy? Economics. v. 44. Institute for Scientific Co-operation. Tubingen, Germany, p. 7-31.

HERRERA, A. et al. 1971 ¿Catrástofe o nueva sociedad? Modelo Mundial Latinoamericano". Fundación Bariloche. CIID. Bogotá.

HOBSBAWM, Eric. 1995. Era dos extremos. O breve século XX, 1914-1991. São Paulo: Companhia das letras.

LÉLÉ, S. M. Sustainable Development: a critical review. World Development. v. 19, n. 6, p. 607-21, jun. Great Britain: Pergamon Press, 1991.

MEADOWS, D;MEADOWS, D; RANDERS, J;BEHRENS, W. Los límites del crecimiento. México D.F.: FCE. 1972.

ONU-EPHE. Organizacion de Naciones Unidas-Ecole practique des Hautes Ètudes 1972. Developement and Environment. La Haye/Paris: Mouton.

PEARCE, David. Blueprint 3. Measuring Sustainable Development. London: Earthscan. 1993.
PEZZOLI, Keith. Sustainable Development: a transdisciplinary overview of the literature. Journal of Environmental Planning and Management. v. 40, n. 5. sept.97.p. 549-574, 1997.

SACHS, Ignacy. Environnement et Styles de Developpement. Annales. v. 3, p. 553-570. 1974.

. Ecodesarrollo. Concepto, aplicación, implicaciones. Comercio Exterior. v. México D. F.: 30, n. 7, p. 718-725. 1980.

Estratégias de transição para o século XXI $\mathrm{Ca}$ dernos de Desenvolvimento e Meio Ambiente, n. 1. Curitiba: Editora UFPR, 1994.

SIMON, J; KAHN, H. The Resourceful Earth. A response to Global 2000. New York: Basil Blackwell, 1984.

SIMMONS, I.G. 1993. Environmental History. Oxford UK, Cambridge USA: Blackwell, 1993.

STRONG, Maurice, 1994. Conferencia de las Naciones Unidas sobre Medio Ambiente y Desarrollo. In: GLENDER, A., LICHTINGER, V. (Comp.). La diplomacia ambiental. México y la Conferencia de las Naciones Unidas sobre Medio Ambiente y Desarrollo. México D.F.: SRE-FCE.

SUNKEL, O; GLIGO, N; KOOLEN, R; BALLESTEROS, R; LAL, J; VIDAL, O; COLLANTES, C; HURTUBIA, J. 1986. La dimensión ambiental en la planificación del desarrollo. Buenos Aires: Grupo Editor Latinoamericano.

SZÉKELY, A; PONCE-NAVA, D. 1994. La declaración de Río y el derecho internacional ambiental. In: GLENDER, A.; LICHTINGER, V. (Comp.). La diplomacia ambiental. México y la Conferencia de las Naciones Unidas sobre Medio Ambiente y Desarrollo. México D. F.: SRE-FCE.

TAMAMES, R. Ecología y Desarrollo. La polémica sobre los límites del crecimiento. Madrid: Alianza Universidad, 1974.

WCED. Our Common Future. Oxford: University Press. 1987. 